\title{
Plasma circulating miR-23 27 24 clusters correlate with the immunometabolic derangement and predict C-peptide loss in children with type 1 diabetes
}

\author{
Silvia Garavelli ${ }^{1,2} \cdot$ Sara Bruzzaniti ${ }^{2,3}$. Elena Tagliabue ${ }^{1} \cdot$ Dario Di Silvestre $^{4} \cdot$ Francesco Prattichizzo $^{1} \cdot$ Enza Mozzillo $^{5}$. \\ Valentina Fattorusso ${ }^{5}$ - Lucia La Sala ${ }^{1}$. Antonio Ceriello ${ }^{1}$. Annibale A. Puca ${ }^{1,6}$. Pierluigi Mauri ${ }^{4}$ - Rocky Strollo ${ }^{7}$. \\ Marco Marigliano ${ }^{8}$. Claudio Maffeis $^{8}$ - Alessandra Petrelli ${ }^{9}$. Emanuele Bosi ${ }^{9,10}$ - Adriana Franzese ${ }^{5}$. \\ Mario Galgani ${ }^{2,11}$ (D) Giuseppe Matarese ${ }^{2,11}$ (D) Paola de Candia ${ }^{1}$
}

Received: 14 April 2020 / Accepted: 15 June 2020 / Published online: 29 July 2020

(C) Springer-Verlag GmbH Germany, part of Springer Nature 2020

\begin{abstract}
Aims/hypothesis We aimed to analyse the association between plasma circulating microRNAs (miRNAs) and the immunometabolic profile in children with type 1 diabetes and to identify a composite signature of miRNAs/immunometabolic factors able to predict type 1 diabetes progression.

Methods Plasma samples were obtained from children at diagnosis of type 1 diabetes $(n=88)$ and at $12(n=32)$ and $24(n=30)$ months after disease onset and from healthy control children with similar sex and age distribution $(n=47)$. We quantified 60 robustly expressed plasma circulating miRNAs by quantitative RT-PCR and nine plasma immunometabolic factors with a recognised role at the interface of metabolic and immune alterations in type 1 diabetes. Based on fasting C-peptide loss over time, children with type 1 diabetes were stratified into the following groups: those who had lost $>90 \%$ of C-peptide compared with diagnosis level; those who had lost $<10 \%$ of C-peptide; those showing an intermediate C-peptide loss. To evaluate the modulation of plasma circulating miRNAs during the course of type 1 diabetes, logistic regression models were implemented and the correlation between miRNAs and immunometabolic factors was also assessed. Results were then validated in an independent cohort of children with recent-onset type 1 diabetes $(n=18)$. The prognostic value of the identified plasma signature was tested by a neural network-based model.

Results Plasma circulating miR-23 27 24 clusters (miR-23a-3p, miR-23b-3p, miR-24-3p, miR-27a-3p and miR-27b-3p) were upmodulated upon type 1 diabetes progression, showed positive correlation with osteoprotegerin (OPG) and were negatively correlated with soluble CD40 ligand, resistin, myeloperoxidase and soluble TNF receptor in children with type 1 diabetes but not
\end{abstract}

Electronic supplementary material The online version of this article (https://doi.org/10.1007/s00125-020-05237-x) contains peer-reviewed but unedited supplementary material, which is available to authorised users.

Paola de Candia

paola.decandia@multimedica.it

Giuseppe Matarese

giuseppe.matarese@unina.it

Mario Galgani

mario.galgan@unina.it

IRCCS MultiMedica, via G. Fantoli 16/15, 20138 Milan, Italy

2 Institute for Endocrinology and Experimental Oncology ' $\mathrm{G}$. Salvatore', C.N.R, via Pansini 5, 80131 Naples, Italy

3 Department of Biology, University of Naples 'Federico II', Naples, Italy

4 Institute of Biomedical Technologies, C. N. R, Segrate, Milan, Italy
5 Centre of Paediatric Diabetology, Department of Translational Medical Sciences, University of Naples 'Federico II', Naples, Italy

6 Department of Medicine and Surgery, University of Salerno, Baronissi, Italy

7 Department of Medicine, Unit of Endocrinology \& Diabetes, Università Campus Bio-Medico, Rome, Italy

8 Paediatric Diabetes and Metabolic Disorders Unit, University of Verona, Verona, Italy

9 San Raffaele Diabetes Research Institute, IRCCS Ospedale San Raffaele, Milan, Italy

10 Vita-Salute San Raffaele University, Milan, Italy

11 Department of Molecular Medicine and Medical Biotechnology, University of Naples 'Federico II', via Pansini 5, 80131 Naples, Italy 


\section{Research in context}

\section{What is already known about this subject?}

- Plasma circulating miRNAs may reflect type 1 diabetes state and progression and are being actively investigated as potential disease biomarkers

What is the key question?

- Do plasma circulating miRNAs mirror type 1 diabetes-associated immunometabolic derangement and are they able to predict disease progression in children with type 1 diabetes?

What are the new findings?

- $\quad$ Plasma circulating miR-23 27 24 clusters (miR-23a-3p, miR-23b-3p, miR-24-3p, miR-27a-3p and miR-27b-3p) increased upon type 1 diabetes progression, and were positively correlated with osteoprotegerin (OPG) and inversely correlated with soluble CD40 ligand, resistin, myeloperoxidase and soluble TNF receptor in children with type 1 diabetes, but not in healthy children

- $\quad$ Parallel assessment of miR-23a-3p, miR-23b-3p, miR-24-3p, miR-27b-3p and OPG at type 1 diabetes onset showed high predictive capability for disease progression (as assessed by C-peptide loss 12 months after disease onset)

How might this impact on clinical practice in the foreseeable future?

- The identified miR-23a-3p/miR-23b-3p/miR-24-3p/miR-27b-3p/OPG panel may be developed into a novel method to better stratify children with type 1 diabetes, thus improving patients' clinical management, assessment of complication risks and selection of the most adequate treatment

in healthy children. The combination of plasma circulating miR-23a-3p, miR-23b-3p, miR-24-3p, miR-27b-3p and OPG, quantified at disease onset, showed a significant capability to predict the decline in insulin secretion 12 months after disease diagnosis in two independent cohorts of children with type 1 diabetes.

Conclusions/interpretations We have pinpointed a novel miR-23a-3p/miR-23b-3p/miR-24-3p/miR-27b-3p/OPG plasma signature that may be developed into a novel blood-based method to better stratify patients with type 1 diabetes and predict C-peptide loss.

Keywords Biomarkers · Cardiovascular risk · Diabetic complications · Immunometabolism · Insulin secretion · microRNA · Osteoprotegerin

$\begin{array}{ll}\begin{array}{l}\text { Abbreviations } \\ \text { cel-miR-39-3p }\end{array} & \begin{array}{l}\text { Caenorhabditis elegans miR-39 } \\ \text { Locked nucleic acid }\end{array} \\ \text { MCP-1 } & \text { Monocyte chemoattractant protein 1 } \\ \text { miRNA } & \text { microRNA } \\ \text { MPO } & \text { Myeloperoxidase } \\ \text { NNM } & \text { Neural network model } \\ \text { OPG } & \text { Osteoprotegerin } \\ \text { ROC } & \text { Receiver operating characteristic } \\ \text { sCD40L } & \text { Soluble CD40 ligand } \\ \text { sICAM } & \text { Soluble intercellular adhesion molecule 1 } \\ \text { sLepR } & \text { Soluble leptin receptor } \\ \text { sTNFR } & \text { Soluble TNF receptor }\end{array}$

\section{Introduction}

Type 1 diabetes is one of the most common chronic autoimmune diseases of childhood in which cells of the innate and adaptive immune system invade the pancreatic islets causing inflammation, beta cell destruction and progressive loss of insulin production [1-4]. When children receive a diagnosis of type 1 diabetes, beta cell mass is already reduced; then, the monitoring of serum concentrations of C-peptide, secreted by beta cells at a one-to-one ratio with insulin, shows that islet loss can be very rapid during the first year of diagnosis [1, 5]. After metabolic stabilisation, only a subgroup of individuals retains the ability to produce insulin; although this endogenous secretion is typically low, its preservation is very important, since it is clinically associated with less severe diabetes-related complications at later stages of disease [6]. There remains an urgent need to identify blood circulating biomarkers able to predict the progression of type 1 diabetes in order to help the overall clinical management for each individual.

A potential source of novel and useful biomarkers is represented by microRNAs (miRNAs), small ( $\sim 22$ nucleotides in length), highly conserved non-coding RNAs [7, 8], which are released at the extracellular level and circulate in blood with 
Table 1 Baseline characteristics of participants

\begin{tabular}{|c|c|c|c|c|c|c|}
\hline \multirow[t]{2}{*}{ Baseline characteristic } & \multicolumn{3}{|l|}{ Discovery cohort } & \multirow{2}{*}{$\begin{array}{l}\text { Validation cohort } 1 \\
\text { Recent-onset T1D }\end{array}$} & \multirow{2}{*}{$\begin{array}{l}\text { Validation cohort } 2 \\
\text { Recent-onset T1D }\end{array}$} & \multirow[t]{2}{*}{$p$ value } \\
\hline & Recent-onset T1D & T1D at 12 months & $\begin{array}{l}\text { T1D at } \\
24 \text { months }\end{array}$ & & & \\
\hline No. of participants & 88 & 32 & 30 & 18 & 26 & - \\
\hline Age, years & $9 \pm 4$ & $12 \pm 4$ & $12 \pm 4$ & $11 \pm 4$ & $10 \pm 4$ & NS \\
\hline Sex, $\%$ male & 54.5 & 56.2 & 53.3 & 66.7 & 76.9 & $\mathrm{NS}^{\mathrm{a}}$ \\
\hline BMI, $\mathrm{kg} / \mathrm{m}^{2}$ & $17.4 \pm 3.4$ & $19.5 \pm 2.9$ & $19.5 \pm 2.8$ & $16.3 \pm 1.9$ & $17.9 \pm 3.7$ & NS \\
\hline C-peptide, nmol/1 & $0.17 \pm 0.13$ & $0.13 \pm 0.13$ & $0.13 \pm 0.20$ & $0.13 \pm 0.07$ & $0.07 \pm 0.03$ & $<0.01$ \\
\hline Ketoacidosis at diagnosis, $n$ yes/no/NA & $35 / 52 / 1$ & $10 / 19 / 3$ & $9 / 13 / 8$ & $9 / 9 / 0$ & $4 / 22 / 0$ & $\mathrm{NS}^{\mathrm{a}}$ \\
\hline $\mathrm{HbA}_{1 \mathrm{c}}, \mathrm{mmol} / \mathrm{mol}$ & $100 \pm 20$ & $61 \pm 18$ & $58 \pm 13$ & $93 \pm 31$ & $104 \pm 26$ & \\
\hline $\mathrm{HbA}_{1 \mathrm{c}}, \%$ & $11.3 \pm 1.8$ & $7.7 \pm 1.7$ & $7.5 \pm 1.2$ & $10.7 \pm 2.9$ & $11.7 \pm 2.4$ & NS \\
\hline Insulin daily dose, $\mathrm{U} / \mathrm{kg}$ & $0.6 \pm 0.3$ & $0.5 \pm 0.3$ & $0.6 \pm 0.3$ & $0.9 \pm 0.5$ & $0.7 \pm 0.3$ & $<0.01$ \\
\hline Other autoimmune disorders, yes/no/NA & $15 / 73 / 0$ & $9 / 20 / 3$ & $6 / 15 / 9$ & $3 / 15 / 0$ & $0 / 26 / 0$ & $\mathrm{NS}^{\mathrm{a}}$ \\
\hline
\end{tabular}

Data are $n, \%$ or mean \pm SEM

Participants were children with type 1 diabetes at recent onset and type 1 diabetes at 12 and 24 months after diagnosis from the discovery cohort and children with type 1 diabetes at recent onset from the two validation cohorts recruited for the study

Statistical analysis was by one-way ANOVA for the three groups of participants at diagnosis

${ }^{\text {a }}$ Statistical analysis was by $\chi^{2}$ test

T1D, type 1 diabetes

high stability. They have already become candidate indicators for a plethora of complex, multifactorial diseases, including both metabolic and autoimmune diabetes [9-24]. In particular, the association studies of circulating miRNAs in type 1 diabetes are heterogeneous in terms of individuals analysed, biological samples evaluated, miRNA detection methods and data normalisation. Notwithstanding some overlapping results, this effort still requires assay standardisation and further replication in multiple cohorts. Moreover, while many studies on type 1 diabetes miRNA signature exist, miRNAs able to predict disease trajectory are not well documented.

The major objective of this work was thus to identify a composite signature of plasma circulating miRNAs and diabetes-relevant immunometabolic factors with potential prognostic value. To this aim, we have retrospectively profiled 60 plasma circulating miRNAs, based on their robust expression, in paediatric individuals with type 1 diabetes. In parallel, we have also profiled a panel of nine immunometabolic factors, set according to their demonstrated role as key signalling molecules at the interface of metabolic alterations and immune cell function in type 1 diabetes [25].

\section{Methods}

\section{Participants}

Diagnosis of type 1 diabetes was defined according to the Global International Diabetes Federation/International Society for Paediatric and Adolescent Diabetes Guidelines for Diabetes in Childhood and included symptoms of diabetes in addition to casual plasma glucose concentration $\geq 11.1 \mathrm{mmol} / 1(200 \mathrm{mg} / \mathrm{dl})$, or fasting plasma glucose $\geq 7.0 \mathrm{mmol} / 1(\geq 126 \mathrm{mg} / \mathrm{dl})$, or $2 \mathrm{~h}$ post-load glucose $\geq 11.1 \mathrm{mmol} / \mathrm{l}(\geq 200 \mathrm{mg} / \mathrm{dl})$ during an OGTT, and $\mathrm{HbA}_{1 \mathrm{c}} \geq 6.5 \%$ (48 $\mathrm{mmol} / \mathrm{mol}$ ) [26]. The baseline characteristics of children with type 1 diabetes enrolled in the present study are reported in Table 1 and the schematic representation of the study is shown in electronic supplementary material (ESM) Fig. 1.

Discovery cohort Children with type 1 diabetes at recent onset $(n=88)$, and $12(n=32)$ and $24(n=30)$ months after diagnosis were recruited at the Department of Translational Medical Sciences, Paediatric section, University of Naples 'Federico II'. The glycaemic control, insulin dose and BMI changes in the follow-up period are shown in ESM Fig. 2a-c. In parallel, healthy control children with similar sex and age distribution $(n=47)$ were recruited in the same Paediatric department, upon selection for the following criteria: fasting blood glucose $<5.5 \mathrm{mmol} / \mathrm{l}$ ( $<100 \mathrm{mg} / \mathrm{dl})$; negative personal and familial history of autoimmune disorders; and negativity for islet autoantibodies at the 99th percentile. The study was approved by the Institutional Review Board (IRB) of the University of Naples 'Federico II', Prot N. 200/16 and parents gave informed consent; assent was obtained from children during the course of the study.

Validation 1 cohort The study was conducted in accordance with local ethics committee approval (IRB no. TIGET004DRI003) and the Declaration of Helsinki at the IRCCS Ospedale San Raffaele, Milan, Italy. Before inclusion in the 
study, written informed consent was obtained either directly from children and/or from parents/guardians; assent was obtained from children during the course of the study. Plasma-EDTA samples from $n=18$ paediatric patients with type 1 diabetes were prepared by two-step centrifugation. All participants showed positivity for one or more autoantibodies at the time of blood collection.

Validation 2 cohort The study was conducted at the Paediatric Diabetes and Metabolic Disorders Unit, University of Verona, Italy and samples were part of the NUTRIMET-VR biobank, in accordance with local ethics committee approval (Prot. N. 47,460). Serum samples were prepared from $n=26$ paediatric individuals with type 1 diabetes by using a serum-separator tube, centrifuged at $1300 \mathrm{~g}$ for $10 \mathrm{~min}$ and stored until use. All participants showed positivity for at least one autoantibody at the time of blood collection. Children with type 1 diabetes from all three cohorts were recruited between 5 and 8 days from disease onset, after blood glucose stabilisation by exogenous insulin (glucose values $3.5-10 \mathrm{mmol} / 1$ [80-180 mg/dl]). Individuals with type 1 diabetes and healthy individuals with recent vaccinations, infections, trauma, oncological pathologies or systemic inflammation were excluded from the study.

\section{Participants' stratification based on C-peptide loss}

For specific analytical purposes, we assigned participants with type 1 diabetes to different groups based on loss of fasting Cpeptide levels occurring 12 months after diagnosis. Our arbitrary stratification was as follows: children who had lost $>90 \%$ of C-peptide compared with diagnosis level; children who had lost $<10 \%$ of C-peptide; children showing an intermediate loss of C-peptide (C-peptide loss between $10 \%$ and $90 \%$ ).

\section{Laboratory testing}

Serum and plasma samples were kept at $-80^{\circ} \mathrm{C}$ until use. Fasting C-peptide levels were measured using a commercial ELISA kit (Millipore Corporation, Germany). Glucose levels were measured using enzymatic hexokinase method and $\mathrm{HbA}_{1 \mathrm{c}}$ by HPLC (HLC-723 G7 TOSOH; Bioscience, Italy).

\section{RNA extraction, heparinase protocol and quantitative RT-PCR}

After thawing, plasma samples were centrifuged at $3000 \mathrm{~g}$ for 5 min at $4{ }^{\circ} \mathrm{C}$ to remove cryoprecipitates and cell debris. Sample haemolysis was measured at $414 \mathrm{~nm}$ (ESM Fig. 2d). Total RNA was isolated from $300 \mu \mathrm{l}$ of plasma in the presence of an MS2 carrier RNA (Roche, Switzerland) using miRCURY RNA Isolation Kit-Biofluid (Qiagen, USA) according to manufacturer's instructions. Plasma was never pooled and RNA samples were prepared from single individuals in all instances. RNA samples from the discovery cohort were heparinase-treated $(0.5 \mathrm{U} / \mu \mathrm{l}$ of isolated RNA) for $1 \mathrm{~h}$ at $25^{\circ} \mathrm{C}$ to recover miRNA detectability (ESM Fig. 2e,f). RNA was then reverse-transcribed with a miRCURY LNA Universal RT Kit and miRNA profiled by quantitative RTPCR, using SYBR Green master mix with locked nucleic acid (LNA)-based miRNA primers (Qiagen). Plates were run on the ABI QuantStudio 6 Flex qPCR machine (the same cycling conditions and parameters were maintained throughout the study). To monitor for RNA isolation efficiency and retrotranscription reproducibility, we routinely spiked-in synthetic RNA-resembling miRNAs (i.e. UniSp2 and UniSp4) to lysis buffer and Caenorhabditis elegans miR-39 (cel-miR-39-3p) to cDNA synthesis, which, by being ultimately measured by RT-qPCR, provided internal references. We profiled 60 miRNAs (miRNA quantitative data available in ESM Table 1) and relative quantitative values were calculated with the $\Delta \mathrm{C}_{\mathrm{t}}$ method, using miRNA global mean (obtained by taking into account the first 30 most-expressed miRNAs per samples, all showing a $C_{t}<35$ ) as a biological normalisation factor (ESM Fig. 2g,h). miRNA quantitative data were plotted as $\log _{2}$ fold change relative to the healthy control cohort.

\section{Inflammatory molecules and cytokine measurement}

Additional plasma samples from the same individuals were thawed and nine immunometabolic factors (leptin, soluble leptin receptor [sLepR], soluble CD40 ligand [sCD40L], osteoprotegerin [OPG], monocyte chemoattractant protein 1 [MCP-1], myeloperoxidase [MPO], soluble intercellular adhesion molecule 1 [sICAM], resistin and soluble TNF receptor [sTNFR]) were analysed using the bead-based multianalyte immunoassay (R\&D Systems, USA) according to the manufacturer's recommendations, and then measured by Multiplex technology (Luminex 200; Luminex, USA); xPONENT 3.1 software (Luminex) was used for data acquisition.

\section{Statistics}

Distribution of continuous variables was tested for normality by the Kolmogorov-Smirnov test. Comparisons between groups were performed by either unpaired Student's $t$ test or non-parametric Mann-Whitney test (for two groups) and by ANOVA or Kruskal-Wallis (for three or more groups) for normally and not-normally distributed variables, respectively. Whisker plots were used to represent distributions of miRNAs, immunometabolic factors and fold changes in miRNA expression. Correlation between miRNAs and immunometabolic factors was graphically represented by scatter plots and heatmaps. To evaluate the association between either participant status (healthy control participants vs type 1 diabetes) or type 1 diabetes progression (high vs low 
C-peptide loss) and collected variables, logistic regression models were implemented. Receiver operating characteristic (ROC) curves were drawn for models, and the corresponding AUCs calculated. Missing values were excluded. Statistical analyses were performed using the SAS software (version 9.4, SAS Institute, Cary, NC, USA) and statistical significance was defined as $p$ value $<0.05$. Given the low number of participants, we were not able to adjust our models of C-peptide loss prediction by BMI, sex and baseline C-peptide. Analysis of differential expression in the discovery cohort was not corrected for multiple testing in order to reduce the risk of ignoring true findings that would have then been independently tested in the validation cohort. Graphical representations were drawn using GraphPad Prism software (version 7, La Jolla, CA USA, www.graphpad.com), with data presented as whisker plots, unless stated otherwise, and with $\mathrm{R}$ software ( $\mathrm{R}$ version 3.4.4; R Foundation for Statistical Computing, Vienna, Austria) by using corrplot library (http://cran.rproject.org/ 15/03/2018).

Neural network model To develop a neural network model (NNM), we processed data by using JMP software version 15 (SAS Institute, Cary, NC, USA). Specifically, TanH activation function (a centred and scaled version of the logistic function) was implemented and a hidden layer with three nodes was applied. Our group of participants was split into a training set (participants from the discovery cohort) and a validation set (participants from validation cohort 1), using the Excluded Row function of JMP. In addition, we used the random Kfold cross-validation method, also implemented in JMP: it is designed for small datasets, divides the original data into $\mathrm{K}$ subsets, in turn used to validate the model fit on the rest of the data, and then selects the best model.

\section{Results}

\section{Plasma circulating miRNA dysregulation during the course of type 1 diabetes}

The present study was divided into two different phases (ESM Fig. 1). During the first study phase, we profiled 60 plasma circulating miRNAs (ESM Table 1), recently selected for being robustly expressed in children's plasma [27], in participants with type 1 diabetes at recent onset $(n=88)$ and upon disease progression (at $12[n=32]$ and $24[n=30]$ months after diagnosis). Baseline characteristics of these participants are shown in Table 1. As a reference, we also quantified these miRNAs in a control group of healthy children with similar sex and age distribution. A high percentage of miRNAs was indeed found to be significantly modulated upon type 1 diabetes progression (27/60 [45\%], ESM Table 2), among which miR-106a-5p, miR-19a-3p, miR-20a-5p, miR-21-5p, miR- 30e-5p, miR-451a and miR-93-5p drew our attention because their type 1 diabetes-dependent dysregulation has also been reported by other laboratories [24, 28-31]. We also noted that three plasma circulating miRNAs remarkably upregulated upon disease progression are members of two highly linked miRNA clusters, collectively called miR-23 27 24 (miR$23 a-3 p$, miR-23b-3p and miR-24-3p), with miR-23a-3p and miR-24-3p in particular almost doubling in children with type 1 diabetes lasting 12 months compared with participants at recent onset (Fig. 1a-c). The other two members of the clusters (miR-27a-3p and miR-27b-3p) showed a similar pattern of plasma increment upon type 1 diabetes progression, although their dysregulation was not statistically significant (Fig. 1d,e). Of note, most of the plasma circulating miRNAs found to be dysregulated upon type 1 diabetes progression, including miR-23 27 24 clusters (18/27 [67\%]), were not significantly affected in children with type 1 diabetes compared with healthy children (ESM Fig. 2i), demonstrating that a distinct plasma miRNA dysregulation is specifically associated with type 1 diabetes progression. Besides one exception (miR-140-3p), none of the plasma circulating miRNAs differentially expressed during the course of type 1 diabetes showed a significant correlation index $(r>0.60)$ with $\mathrm{HbA}_{1 \mathrm{c}}$ (ESM Table 3), suggesting that miRNA modulation may be linked to autoimmune disease progression, rather than to hyperglycaemia.

\section{Plasma immunometabolic dysregulation during the course of type 1 diabetes}

In the same plasma samples analysed for miRNA quantities, we also tested nine immunometabolic factors (ESM Table 4), in order to register the immunometabolic derangement that accompanied type 1 diabetes onset and progression in these children. Indeed, as a result of the known metabolic decompensation associated with dramatic changes of the leptin axis $[25,32,33]$, levels of sLepR were found to escalate in children with type 1 diabetes compared with healthy children (Fig. 1f). We also found an increase in plasma OPG and a reduction in MPO in children with recent-onset type 1 diabetes (Fig. 1g,h). Levels of sLepR, OPG and MPO changed during type 1 diabetes progression and at 24 months after disease onset had returned to measurements similar to those registered in healthy children (Fig. 1f-h).

\section{Elevated levels of plasma circulating miR-23 27 24 clusters associate with C-peptide loss in children with type 1 diabetes}

To evaluate whether plasma circulating miRNAs may distinguish children with different type 1 diabetes progression rate, we analysed miRNA expression levels (as registered at onset) upon stratification of children according to insulin secretion 

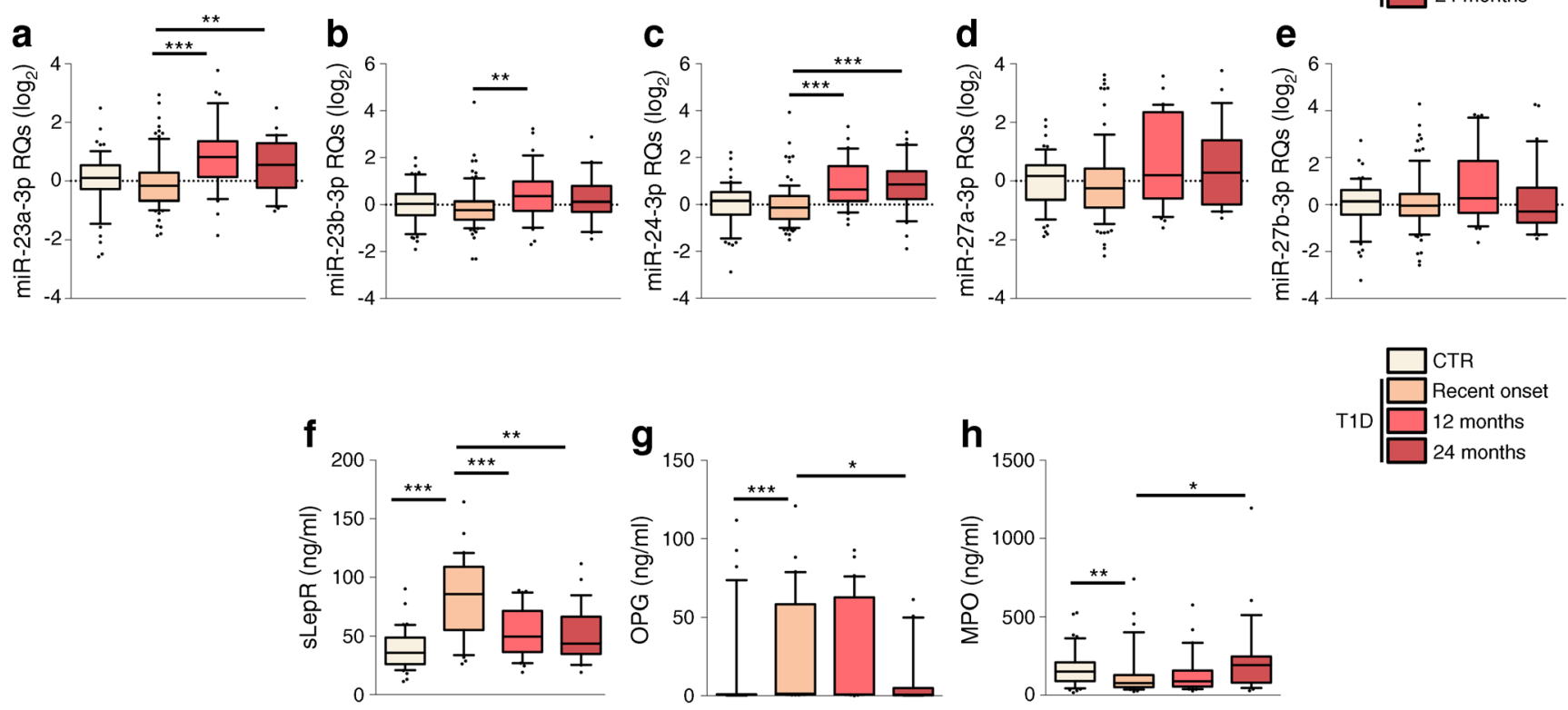

Fig. 1 Plasma circulating miRNA and immunometabolic modulation upon type 1 diabetes progression. Whisker plots (boxes, interquartile range; horizontal lines within boxes, median; whiskers, 10th-90th percentile) showing $\log _{2}$ relative quantity of plasma circulating miR23 27 24 clusters (a-e) and plasma levels of sLepR (f), OPG (g) and MPO (h) in children with type 1 diabetes at onset and upon disease progression (12 and 24 months after diagnosis). A control group of healthy children is included as reference. Statistical analysis was performed by unpaired Student's $t$ test or by Mann-Whitney test

loss (measured as fasting C-peptide levels) occurring 12 months after disease onset. Specifically, children with type 1 diabetes were arbitrarily divided into the following groups: (1) those who had lost $>90 \%$ of C-peptide compared with diagnosis level; (2) those who had lost $<10 \%$ of C-peptide; and (3) those with an intermediate Cpeptide loss (10-90\%). The three groups were not significantly different in terms of C-peptide at onset of diabetes (Fig. 2a). The first group was formed by younger children (although the difference was not statistically significant), while $\mathrm{HbA}_{1 \mathrm{c}}$ did not differ among the three groups (ESM Fig. 3a,b). Among the 60 miRNAs profiled in these participants, 17 were differentially expressed in the two extreme groups ( $>90 \%$ vs $<10 \%$ C-peptide loss) of children with type 1 diabetes: expression levels of six were lower while expression levels of 11 were greater in children with $>90 \%$ C-peptide loss (Fig. 2b). MiR-223-3p was significantly anti-correlated with children's age at onset, suggesting that its higher expression in children who later showed $>90 \%$ C-peptide loss may be dependent on this association (ESM Fig. 3c). Beside miR-223-3p, the five miRNAs showing the greatest difference in mean expression at diabetes onset for children with $>90 \%$ vs $<10 \%$ C-peptide loss were miR- depending on data distribution. For miRNAs, $n=47$ healthy children (CTR), $n=88$ children with recent-onset diabetes, $n=32$ children with diabetes of 12 months' duration and $n=30$ children with diabetes of 24 months' duration. For immunometabolic factors, $n=43$ healthy children (CTR), $n=32$ children with recent-onset type 1 diabetes, $n=30$ children with diabetes of 12 months' duration and $n=25$ children with diabetes of 24 months' duration. ${ }^{*} p<0.05, * * p<0.01$ and $* * * p<0.001$. $\mathrm{CTR}$, healthy children; RQ, relative quantity; T1D, type 1 diabetes

23 27 24 clusters, already identified as escalating during the course of the disease (Fig. 2c-g). The children with intermediate C-peptide loss at 12 months after type 1 diabetes diagnosis showed intermediate differences in these miRNAs at disease onset. MiR-23 27 24 clusters did not show any meaningful association with age at type 1 diabetes onset (ESM Fig. 3d-h) or with C-peptide level at baseline (ESM Fig. 3i-m).

To overcome the difficulty of using the miRNA global mean (assessed through the quantification of tens of different miRNAs), we tested the association between C-peptide loss and miRNA ratio (i.e. the $C_{t}$ difference $\left[\Delta C_{t}\right]$ of couples of miRNAs, composed of the five members of the miR23 27 24 clusters as numerators). As useful potential denominators, we used the six miRNAs with significantly lower expression levels in children exhibiting $>90 \%$ vs $<10 \%$ Cpeptide loss (let-7g-5p, let-7d-3p, miR-425-5p, miR-181a, miR-221-3p and miR-1260a) (Fig. 2b). Among these, miR1260a was the best denominator since it was able to maintain the differential expression of all five miR-23 27 24 cluster members between the two groups of children (Fig. $2 \mathrm{~h}-1$ ), also evaluated by a multivariate analysis adjusted for children's age (ESM Table 5). 

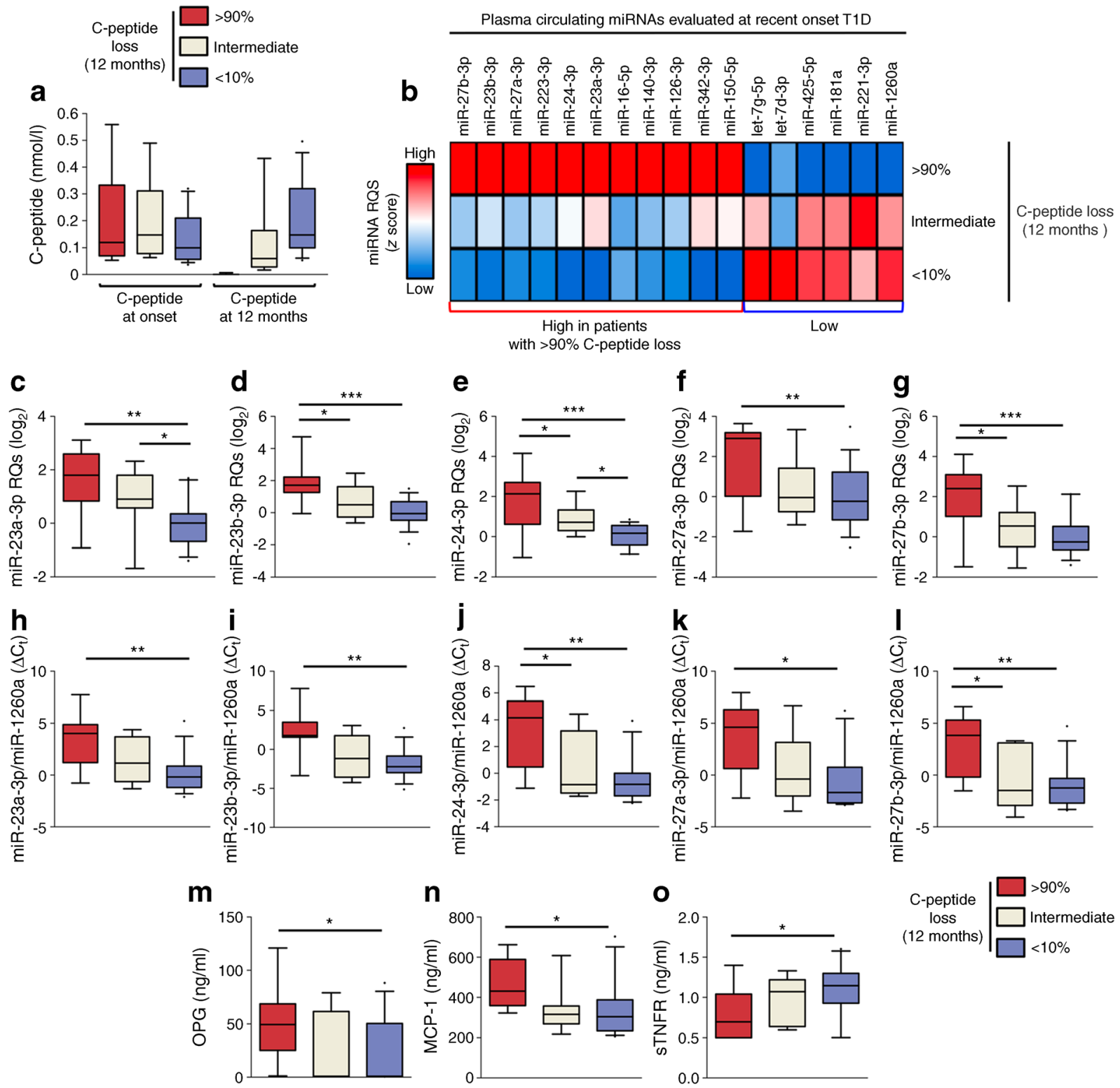

Fig. 2 Plasma circulating miR-23 27 24 clusters, OPG, MCP-1 and sTNFR quantified at type 1 diabetes onset, associated with C-peptide loss occurring 12 months after diagnosis. (a) Whisker plot showing fasting C-peptide registered at baseline and at 12 months after type 1 diabetes onset in the same participants with the indicated C-peptide loss. (b) Heatmap showing the average expression level of plasma circulating miRNAs at type 1 diabetes onset identified to be differentially expressed in children with type 1 diabetes who, 12 months later, exhibited $>90 \%$ vs $<10 \%$ C-peptide loss. miRNAs are ordered based on $\log _{2}$ fold change between the two groups of children. (c-I) Whisker plots showing $\log _{2}$ relative quantity of plasma circulating miR23 27 24 clusters at diagnosis of type 1 diabetes, normalised by either

Children who displayed $>90 \%$ C-peptide loss at 12 months post diabetes diagnosis (vs $<10 \%$ C-peptide loss) were also characterised by higher levels of plasma OPG and MCP-1, and lower levels of sTNFR, at diabetes onset (Fig. $2 \mathrm{~m}-\mathrm{O}$ ). As observed for miRNAs, the group of children displaying intermediate Cpeptide loss 12 months after diabetes diagnosis showed intermediate differences in OPG, MCP-1 and sTNFR at disease onset.
miRNA global mean $(\mathbf{c}-\mathbf{g})$ or miR-1260a $\left(\Delta \mathrm{C}_{t}\right)(\mathbf{h}-\mathbf{l})$ in children who showed $>90 \%,<10 \%$ and intermediate C-peptide loss 12 months after diagnosis. (mo) Plasma concentration, at diagnosis of type 1 diabetes, of OPG (m), MCP-1 (n) and sTNFR (o) in children who showed $>90 \%,<10 \%$ and intermediate Cpeptide loss at 12 months after diagnosis. Whisker plots: boxes, interquartile range; horizontal lines within boxes, median; whiskers, 10th-90th percentile. Statistical analysis was performed by unpaired Student's $t$ test or by MannWhitney test depending on data distribution. $n=8$ children with $>90 \%$ Cpeptide loss, $n=9$ children with intermediate C-peptide loss and $n=15$ children with $<10 \%$ C-peptide loss. ${ }^{*} p<0.05,{ }^{* *} p<0.01$ and $* * * p<0.001$ (twotailed). RQ, relative quantity; T1D, type 1 diabetes

\section{Significant correlation of plasma circulating miR- 23 27 24 clusters with the immunometabolic derangement in children with type 1 diabetes}

We then decided to investigate whether a mutual relationship exists between plasma circulating miR-23 27 24 clusters and the immunometabolic factors (not only OPG, MCP-1 and 


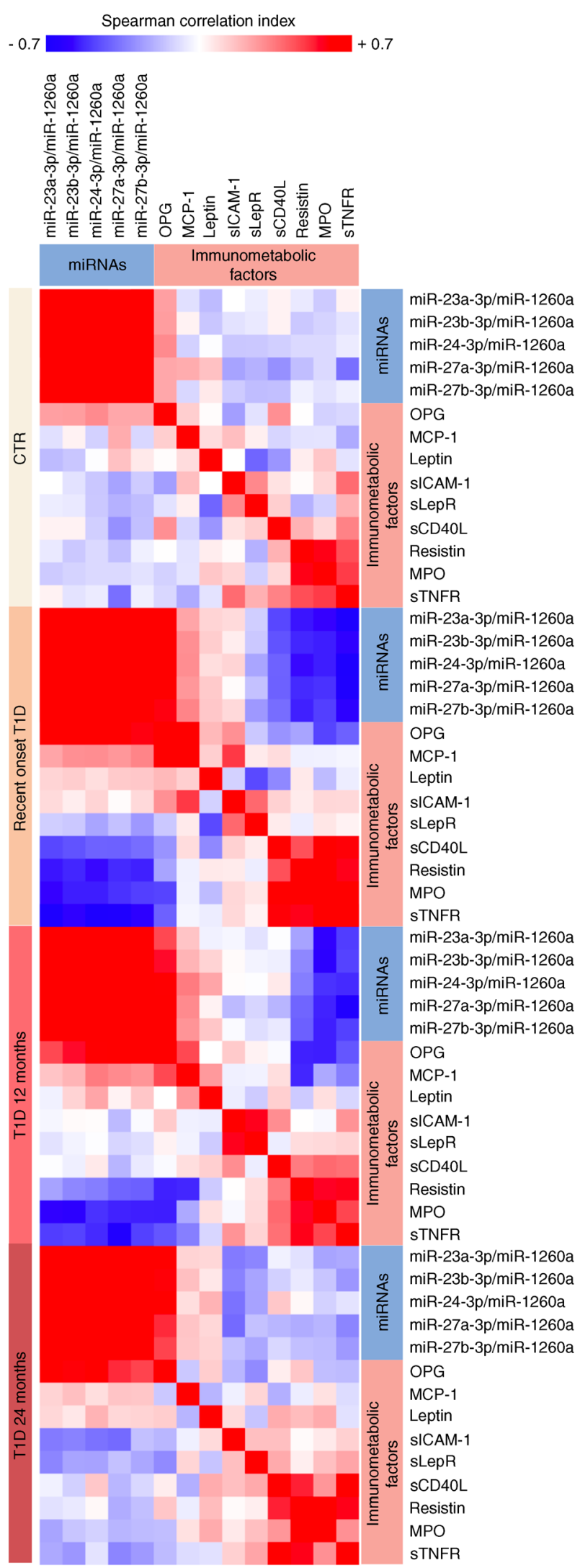

sTNFR but also the other factors tested) and, if so, whether this relationship depends on disease status/progression. To
Fig. 3 Correlation between plasma miR-23 27 24 clusters and immunometabolic factors in children with type 1 diabetes and in healthy children. Heatmap showing the correlation matrix among the indicated plasma circulating miR-23 27 24 cluster members (normalised by miR-1260a) and plasma levels of immunometabolic factors in healthy children and in children with type 1 diabetes at recent onset and 12 and 24 months after diagnosis as indicated. Correlation between two variables is expressed by means of a red (positive) or blue (negative) colour and its intensity is proportional to the Spearman correlation index (scale is reported). Spearman $r$ and relative $p$ values are reported in ESM Table 6. CTR, healthy children; T1D, type 1 diabetes

this aim, we analysed the correlation between the levels of miR-23 27 24 clusters (in relation to miR-1260a) and protein levels in children with type 1 diabetes over time, in comparison with the control group of healthy children (Fig. 3). While miRNA-miRNA correlation was markedly high in all conditions, the protein-protein and miRNA-protein correlation pattern dramatically changed in the different groups of children. Type 1 diabetes status polarised a positive association between SCD40L, resistin, MPO and sTNFR and an inverse correlation between these factors and miR-23 27 24 clusters. Furthermore, miR-23 27 24 clusters were directly and significantly associated with OPG in children with type 1 diabetes at all time points. This complex pattern seems to be dependent on the immunometabolic derangement in type diabetes conditions since none of these associations was observed in healthy children (Fig. 3 and ESM Table 6).

\section{Validation of plasma circulating miR-23 27 24 clusters and OPG association with loss of insulin secretion in an independent cohort of children with type 1 diabetes}

To corroborate our results, in the second study phase (ESM Fig. 1), we tested the identified plasma circulating miRNA and immunometabolic signature in a new independent cohort of individuals with recent-onset type 1 diabetes $(n=18)$, followed in a hospital located in a different geographical area (validation cohort 1; baseline characteristics reported in Table 1). Notably, four out of five miR-23 27 24 cluster members (miR-23a-3p, miR-23b-3p, miR-24-3p and miR$27 b-3 p$ [normalised by miR-1260a]), were confirmed to be present at significantly higher levels, at diagnosis, in plasma of children showing $>90 \%$ vs $<10 \%$ C-peptide loss after 12 months (Fig. 4a-e). Moreover, while changes in concentrations of MCP-1 did not reproduce those observed in the discovery cohort (data not shown), OPG was confirmed to be elevated in children with $>90 \%$ vs $<10 \%$ C-peptide loss in this new cohort (Fig. 4f). Furthermore, the positive correlation between miR-23a-3p, miR-23b-3p, miR-24-3p and miR$27 b-3 p$ and OPG registered in the discovery cohort, was verified in these independent new samples (Fig. 4g-k). 

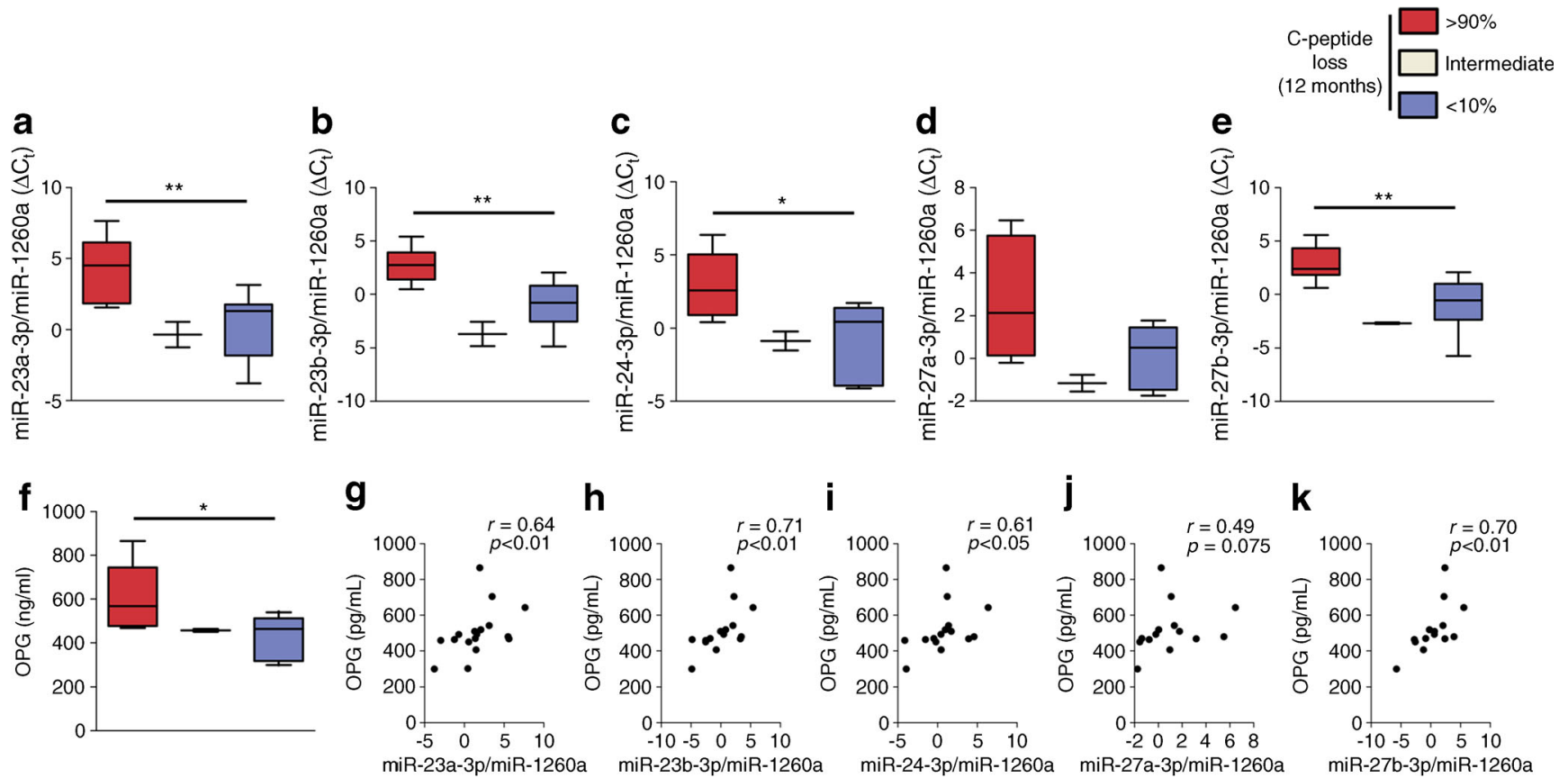

Fig. 4 Validation of miR-23 27 24 clusters and OPG as associated with C-peptide loss upon type 1 diabetes progression in an independent cohort of patients. (a-f) Whisker plots (boxes, interquartile range; horizontal lines within boxes, median; whiskers, 10th-90th percentile) showing the level, at diagnosis, of the miR-23 27 24 clusters normalised by miR-1260a $\left(\Delta C_{t}\right)(\mathbf{a}-\mathbf{e})$ and plasma concentration of OPG $(\mathbf{f})$ in children with type 1 diabetes displaying the indicated C-peptide loss 12 months after disease onset. Statistical analysis was performed by unpaired
Student's $t$ test or by Mann-Whitney test depending on data distribution. $n=6$ children with $>90 \%$ C-peptide loss, $n=2$ children with intermediate C-peptide loss and $n=10$ children with $<10 \%$ C-peptide loss. $* p<0.05$ and $* * p<0.01$ (one-tailed). (g-k) Scatter plots showing the one-to-one correlations between plasma levels of OPG and circulating miR23 27 24 clusters. Eighteen $x-y$ pairs were analysed. Spearman $r$ and $p$ values are also shown

plasma circulating miR-23a-3p, miR-23b-3p, miR-24-3p and miR-27b-3p (all normalised by miR-1260a) and plasma level of OPG at disease onset (Fig. 5a). We used quantification data from 31 children who displayed either $>90 \%(n=13)$ or $<10 \%$ $(n=18)$ C-peptide loss at 12 months after type 1 diabetes onset (from both our experimental discovery and validation 1 cohorts). In one approach, the participants from the discovery cohort (seven and 12 individuals with $>90 \%$ and $<10 \% \mathrm{C}$ peptide loss, respectively) were used for NNM training and subsequently performance of the NNM was assessed in 12 individuals from the validation cohort 1 (six and six individuals with $>90 \%$ and $<10 \%$ C-peptide loss, respectively), by using the 'excluded row' method. By this approach, the sample subdivision in training and validation set for the NNM was dictated by the actual experimental approach and either $17 / 19(89 \%)$ or $10 / 12$ individuals $(83 \%)$ from the training and validation set, respectively, were correctly classified as displaying a $>90 \%$ or $<10 \%$ C-peptide loss, respectively (Fig. 5b,c). In a second approach, we implemented the random Kfold method, that arbitrarily divides the sample collection into a training and a validation set and chooses the best fitting model. In this second scenario, the predictive capability of the NNM within the fully trained dataset was 0.94 , while that on unseen data reached 1.0, with all samples (6/6) being correctly classified (Fig. 5d,e). 
a

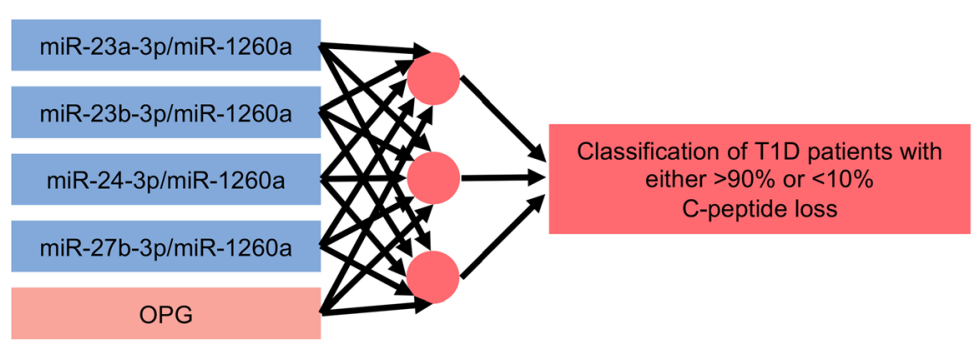

b

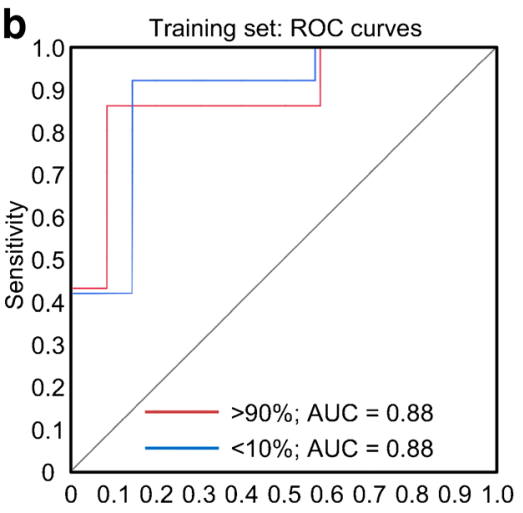

C Validation set: ROC curves

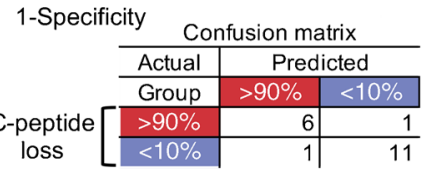

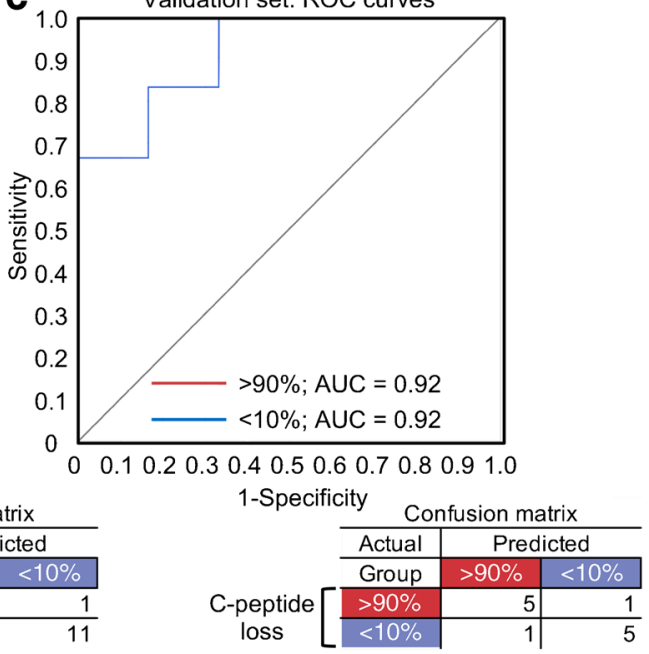

d

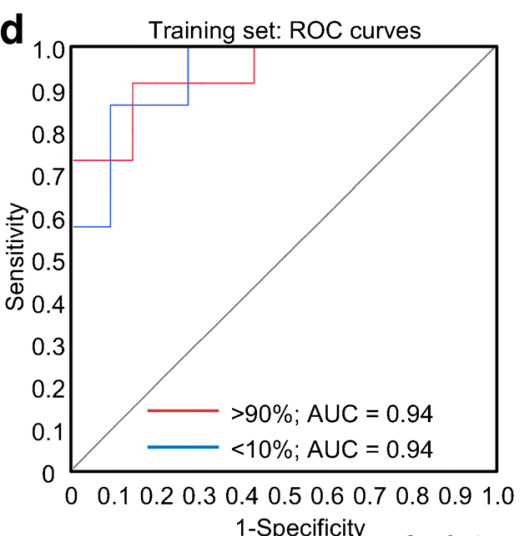

e Validation set: ROC curves

\begin{tabular}{|c|c|c|c|}
\hline & \multicolumn{3}{|c|}{ nTu } \\
\hline & Actual & \multicolumn{2}{|c|}{ Predicted } \\
\hline & Group & $>90 \%$ & $<10 \%$ \\
\hline C-peptide & $>90 \%$ & 9 & 2 \\
\hline loss & $<10 \%$ & 2 & 12 \\
\hline
\end{tabular}

00.10 .20 .30 .40 .50 .60 .70 .80 .91 .0

1-Specificity

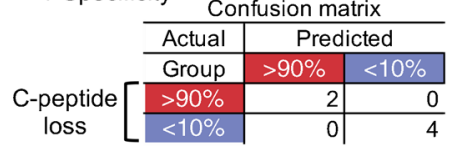

Fig. $5 \mathrm{NNM}$ to evaluate the combination of miR-23a-3p, miR-23b-3p, miR-24-3p, miR-27b-3p and OPG at type 1 diabetes (T1D) onset as predictor of C-peptide loss over time. (a) Inputs to the NNM included plasma circulating miR-23a-3p, miR-23b-3p, miR-24-3p and miR-27b$3 p$ (all normalised by miR-1260a) and plasma level of OPG at onset of type 1 diabetes in children with high $(>90 \%, n=13)$ and low $(<10 \%, n=$ 18) loss of C-peptide, 12 months after disease onset (participants from both our experimental discovery and validation 1 cohorts). A hidden layer with three nodes was applied. (b, c) ROC curves showing the capability of the five input parameters shown in (a) to predict type 1 diabetes with either $>90 \%$ or $<10 \%$ C-peptide loss (evaluated 12 months after diagnosis) for the fully trained dataset (7 with high and 12 with low loss, b) and for the validation dataset ( 6 with high and 6 with low loss, c) by the 'excluded row' method. Confusion matrixes reporting the actual and the predicted classification of high and low C-peptide loss during both the training and the validation test are also reported. (d, e) ROC curves showing the capability of the five input parameters as shown in (a) to predict $\mathrm{C}$ peptide loss (evaluated 12 months after diagnosis) for the fully trained dataset (11 with $>90 \%$ and 14 with $<10 \%$ loss, d) and for the validation dataset ( 2 with $>90 \%$ and 4 with $<10 \%$ loss, e) by the 'random Kfold' method. Confusion matrixes reporting the actual and the predicted classification of high and low C-peptide loss during both the training and the validation test are also reported. The best of five random models was selected 


\section{Discussion}

The connection between plasma circulating miRNAs and the immunometabolic derangement that characterises the pathogenesis of type 1 diabetes is poorly explored. Our effort allowed us to unveil a correlation pattern distinctive of type 1 diabetes status, that comprises miR-23 27 24 clusters, OPG, sCD40L, resistin, MPO and sTNFR. Moreover, we have also discovered that the co-modulated signature composed of four miR-23 27 24 cluster members (miR$23 a-3 p, \operatorname{miR}-23 b-3 p, \operatorname{miR}-24-3 p$ and miR-27b-3p) and OPG, quantified at type 1 diabetes onset, is able to predict the faster decline of insulin secretion 1 year later.

MiR-23 27 24 clusters reside in two genomic loci in humans and are transcribed in a coordinated manner as polycistronic units that are processed to produce individual miRNAs, resulting in co-expression patterns. While the overall behaviour of coexpressed families of miRNAs in human plasma remains largely undiscovered, here we have highlighted a coordinated modulation of miR-23 27 24 clusters that may connect in a cooperative manner to affect the fate of systemic pathways. In this regard, it is noteworthy that members of the miR-23 27 24 clusters have been previously associated with the metabolic syndrome, pancreatic beta cell dysregulation and diabetic complications [20, 34-38]. Moreover, gene repression by miR-23 27 24 clusters is key in the fine tuning of proper $\mathrm{T}$ cell biology and they are released in the extracellular space upon $\mathrm{T}$ cell activation $[39,40]$. In particular, miR-23 27 24 clusters were shown to contribute to unrestricted expansion of diabetogenic cytotoxic $\mathrm{T}$ cells and $\mathrm{T}$ helper cytokine secretion, sustaining a direct relationship between miR-23 27 24 clusters and the typical proinflammatory network of type 1 diabetes [41-43].

The established normalisation strategy required to compare plasma miRNA expression values obtained by PCR quantification is based on the use of the average value of all the expressed miRNAs [44]. To avoid the quantification of this large number of miRNAs for every sample, we have here implemented the analysis of two miRNA reciprocal quantities (miRNA ratios) that, by providing an intrinsically relative value, does not require an additional normalisation procedure. This strategy has been used previously in the search for lung cancer biomarkers [45] but here it is used for the first time in the context of diabetes. The comparable quantities of miRNA ratios that we obtained from samples with different geographical origins, collection, processing and storage procedures show the universal validity of this approach and will enable further inter-laboratory experimental efforts, extremely useful for validation of disease biomarkers.

To our knowledge, this study is the first to correlate plasma level of specific cell-free miRNA ratios with a number of immunometabolic factors, such as $\mathrm{SCD} 40 \mathrm{~L}$, resistin, MPO and sTNFR, already found to be associated with inflammation and beta cell function [46-49]. In particular, miR-23 27 24 clusters positively correlated with OPG, also known as TNF receptor superfamily, member $11 \mathrm{~b}$, a member of the TNFrelated family implicated in the process of bone remodelling [50-52]. Importantly, OPG is involved in the pathogenesis of a plethora of diabetic complications: retinopathy; angiopathy; low bone mineral density with lower osteoblast and increased osteoclast signalling, predisposing to adult osteopenia and osteoporosis; extraosseous calcification, resulting in stiffening of the arteries and subsequent vascular insufficiency; nephropathy; and general cardiovascular morbidity in children with type 1 diabetes [53-60].

Despite the observed alteration in circulating miRNA levels and immunometabolic factors possibly being instigated by either the metabolic or the inflammatory imbalance, our results fit in a framework where this composite signature of molecules and their complex interrelation may function as a rheostat of the heterogeneous (i.e. metabolic and immune) pathogenetic nature of type 1 diabetes. We have very recently demonstrated a link between a novel $\mathrm{CD} 3{ }^{+} \mathrm{CD} 56^{+}$regulatory $\mathrm{T}$ cell population and disease progression in individuals with type 1 diabetes [61] but a tractable biomarker to trace loss of immunological tolerance during disease progression is still missing. Hence, besides the potential pathogenetic involvement of miR23 27 24 clusters, our results demonstrating their predictive capability are clinically relevant. Our results also indicate that the prognostic value of miR-23a-3p, miR-23b-3p, miR-24-3p and miR-27b-3p may reside in plasma but not serum samples, possibly because of extracellular miRNA level perturbation as a result of the coagulation process linked to serum collection (as already hypothesised [62]). More studies are needed to better clarify the strict necessity for plasma usage.

We have concentrated our attention on 60 miRNAs based on robust expression but do acknowledge that there may exist other miRNAs with lower expression levels but higher prognostic value that were not examined. Another limitation of the study resides in the low number of participants with type 1 diabetes; nonetheless, we achieved validation of our results in an independent cohort, thus supporting the identified miR-23a3p/miR-23b-3p/miR-24-3p/miR-27b-3p/OPG combined panel as a potential novel plasma-based method to better stratify patients with type 1 diabetes. Also, we highlighted a complex systemic epigenetic/immunometabolic rearrangement anticipating type 1 diabetes progression, whose a [i.e. aetiopathological] etiopathological relevance deserves further exploration in the future.

Acknowledgements We acknowledge A. Stabilini (San Raffaele Diabetes Research Institute, Milan, Italy) who performed sample collection, processing, storage and recovery and R. Bonfanti (Paediatric Department, Ospedale San Raffaele, Milan, Italy) who was involved in the enrolment of participants from validation cohort 1 . We would also like to thank A. Perfetti and F. Martelli (IRCCS Policlinico San Donato, Milan, Italy) for sharing the heparinase protocol. Schematic figures (including those in the ESM) were created with images adapted from Servier Medical Art by Servier (http://www.servier.fr/servier-medical- 
art). Finally, we express gratitude to A. Pontiroli (Health Science Department, University of Milan, Italy) and S. Scarpetta (Physics Department, University of Salerno, Italy) for useful discussions and to A. Torri for her precious contribution in setting up the plasmatic miRNA quantification.

Data availability All data generated or analysed during this study are included in this published article (and its supplementary information files). In particular, miRNA quantitative data are available in ESM Table 1.

Funding PdC gratefully acknowledges funding from the JDRF for this research (JDRF no. 1-SRA-2018-477-S-B). PdC is also funded by Fondazione Italiana Sclerosi Multipla (FISM no. 2016/R/10 and n. 2018/R/4). This work was also supported by JDRF grant no. 2-SRA2018-479-S-B to MG and by funds from the European Foundation for the Study of Diabetes/Juvenile Diabetes Research Foundation/Lilly program 2015 to GM and program 2016 to MG. MG was also funded by the National Multiple Sclerosis Society (NMSS no. PP-1804-30725) and GM was also funded by FISM (grant 2016/R/18 and 2018/S/5) and Progetti di Rilevante Interesse Nazionale (PRIN) 2017 K55HLC 001. AP is supported by JDRF grant no. 3-APF-2019-744-A-N. This work has also been supported by Italian Ministry of Health Ricerca Corrente IRCCS MultiMedica.

Duality of interest The authors declare that there are no relationships or activities that might bias, or be perceived to bias, their work.

Contribution statement PdC designed the study. AF, EM and VF were responsible for the recruitment of, and related clinical data collection from, type 1 diabetes and healthy control paediatric participants belonging to the discovery cohort. SB and MG were responsible for specimen handling and storage. AP and EB were responsible for the recruitment of, and related collection of clinical data from, type 1 diabetes paediatric participants belonging to validation cohort 1. MM and CM were responsible for the recruitment of, and collection of related clinical data from, type 1 diabetes paediatric participants belonging to validation cohort 2 . SG performed the microRNA profiling and SB the immunometabolic factor quantification. PdC, ET and DDS conducted statistical and bioinformatic analyses. DDS also performed the NNM. FPra, RS, LLS, AC, AAP and PM helped with data analysis and evaluation and critically revised the work for its intellectual content. PdC, MG and GM concurred on final data interpretation and manuscript writing, and all authors contributed to its editing and gave final approval of the version to be published. PdC accepts full responsibility for the work and/or the conduct of the study, had access to the data, and controlled the decision to publish.

\section{References}

1. Eisenbarth GS (1986) Type I diabetes mellitus. A chronic autoimmune disease. N Engl J Med 314(21):1360-1368. https://doi.org/ 10.1056/NEJM198605223142106

2. Gale EA (2005) Type 1 diabetes in the young: the harvest of sorrow goes on. Diabetologia 48(8):1435-1438. https://doi.org/10.1007/ s00125-005-1833-0

3. Moltchanova EV, Schreier N, Lammi N, Karvonen M (2009) Seasonal variation of diagnosis of type 1 diabetes mellitus in children worldwide. Diabet Med 26(7):673-678. https://doi.org/10. 1111/j.1464-5491.2009.02743.x

4. Kolb H, Kolb-Bachofen V, Roep BO (1995) Autoimmune versus inflammatory type I diabetes: a controversy? Immunol Today 16(4):170-172. https://doi.org/10.1016/0167-5699(95)80115-4
5. Greenbaum CJ, Beam CA, Boulware D et al (2012) Fall in Cpeptide during first 2 years from diagnosis: evidence of at least two distinct phases from composite Type 1 Diabetes TrialNet data. Diabetes 61(8):2066-2073. https://doi.org/10.2337/db11-1538

6. Steffes MW, Sibley S, Jackson M, Thomas W (2003) $\beta$-Cell function and the development of diabetes-related complications in the diabetes control and complications trial. Diabetes Care 26(3):832836. https://doi.org/10.2337/diacare.26.3.832

7. Bartel DP (2018) Metazoan microRNAs. Cell 173(1):20-51. https://doi.org/10.1016/j.cell.2018.03.006

8. Friedman RC, Farh KK, Burge CB, Bartel DP (2009) Most mammalian mRNAs are conserved targets of microRNAs. Genome Res 19(1):92-105. https://doi.org/10.1101/gr.082701.108

9. Chen X, Ba Y, Ma L et al (2008) Characterization of microRNAs in serum: a novel class of biomarkers for diagnosis of cancer and other diseases. Cell Res 18(10):997-1006. https://doi.org/10.1038/cr. 2008.282

10. Arroyo JD, Chevillet JR, Kroh EM et al (2011) Argonaute2 complexes carry a population of circulating microRNAs independent of vesicles in human plasma. Proc Natl Acad Sci U S A 108(12):5003-5008. https://doi.org/10.1073/pnas.1019055108

11. Mitchell PS, Parkin RK, Kroh EM et al (2008) Circulating microRNAs as stable blood-based markers for cancer detection. Proc Natl Acad Sci U S A 105(30):10513-10518. https://doi.org/ 10.1073/pnas.0804549105

12. Boeri M, Verri C, Conte D et al (2011) MicroRNA signatures in tissues and plasma predict development and prognosis of computed tomography detected lung cancer. Proc Natl Acad Sci U S A 108(9):3713-3718. https://doi.org/10.1073/pnas.1100048108

13. Moussay E, Wang K, Cho JH et al (2011) MicroRNA as biomarkers and regulators in B-cell chronic lymphocytic leukemia. Proc Natl Acad Sci U S A 108(16):6573-6578. https://doi.org/10. 1073/pnas.1019557108

14. Taylor DD, Gercel-Taylor C (2008) MicroRNA signatures of tumor-derived exosomes as diagnostic biomarkers of ovarian cancer. Gynecol Oncol 110(1):13-21. https://doi.org/10.1016/j. ygyno.2008.04.033

15. D'Alessandra Y, Devanna P, Limana F et al (2010) Circulating microRNAs are new and sensitive biomarkers of myocardial infarction. Eur Heart J 31(22):2765-2773. https://doi.org/10.1093/ eurheartj/ehq167

16. Goretti E, Vausort M, Wagner DR, Devaux Y (2013) Association between circulating microRNAs, cardiovascular risk factors and outcome in patients with acute myocardial infarction. Int J Cardiol 168(4):4548-4550. https://doi.org/10.1016/j.ijcard.2013.06.092

17. Starkey Lewis PJ, Dear J, Platt V et al (2011) Circulating microRNAs as potential markers of human drug-induced liver injury. Hepatology 54(5):1767-1776. https://doi.org/10.1002/hep. 24538

18. Chen YJ, Zhu JM, Wu H et al (2013) Circulating microRNAs as a fingerprint for liver cirrhosis. PLoS One 8(6):e66577. https://doi. org/10.1371/journal.pone.0066577

19. Bartels CL, Tsongalis GJ (2009) MicroRNAs: novel biomarkers for human cancer. Clin Chem 55(4):623-631. https://doi.org/10.1373/ clinchem.2008.112805

20. Karolina DS, Tavintharan S, Armugam A et al (2012) Circulating miRNA profiles in patients with metabolic syndrome. J Clin Endocrinol Metab 97(12):E2271-E2276. https://doi.org/10.1210/ jc.2012-1996

21. Guay C, Regazzi R (2013) Circulating microRNAs as novel biomarkers for diabetes mellitus. Nat Rev Endocrinol 9(9):513521. https://doi.org/10.1038/nrendo.2013.86

22. Zampetaki A, Kiechl S, Drozdov I et al (2010) Plasma microRNA profiling reveals loss of endothelial miR-126 and other microRNAs in type 2 diabetes. Circ Res 107(6):810-817. https://doi.org/10. 1161/CIRCRESAHA.110.226357 
23. Schmittgen TD, Lee EJ, Jiang J et al (2008) Real-time PCR quantification of precursor and mature microRNA. Methods 44(1):3138. https://doi.org/10.1016/j.ymeth.2007.09.006

24. Dotta F, Ventriglia G, Snowhite IV, Pugliese A (2018) MicroRNAs: markers of $\beta$-cell stress and autoimmunity. Curr Opin Endocrinol Diabetes Obes 25(4):237-245. https://doi.org/ 10.1097/MED.0000000000000420

25. Galgani M, Nugnes R, Bruzzese D et al (2013) Metaimmunological profiling of children with type 1 diabetes identifies new biomarkers to monitor disease progression. Diabetes 62(7): 2481-2491. https://doi.org/10.2337/db12-1273

26. Craig ME, Hattersley A, Donaghue KC (2009) Definition, epidemiology and classification of diabetes in children and adolescents. Pediatr Diabetes 10(Suppl 12):3-12. https://doi.org/10.1111/j. 1399-5448.2009.00568.x

27. Garavelli S, Bruzzaniti S, Tagliabue E et al (2020) Blood cocirculating extracellular microRNAs and immune cell subsets associate with type 1 diabetes severity. Int J Mol Sci 21(2):477. https:// doi.org/10.3390/ijms21020477

28. Akerman L, Casas R, Ludvigsson J, Tavira B, Skoglund C (2018) Serum miRNA levels are related to glucose homeostasis and islet autoantibodies in children with high risk for type 1 diabetes. PLoS One 13(1):e0191067. https://doi.org/10.1371/journal.pone. 0191067

29. Seyhan AA, Nunez Lopez YO, Xie H et al (2016) Pancreasenriched miRNAs are altered in the circulation of subjects with diabetes: a pilot cross-sectional study. Sci Rep 6:31479. https:// doi.org/10.1038/srep31479

30. Snowhite IV, Allende G, Sosenko J, Pastori RL, Messinger Cayetano S, Pugliese A (2017) Association of serum microRNAs with islet autoimmunity, disease progression and metabolic impairment in relatives at risk of type 1 diabetes. Diabetologia 60(8): 1409-1422. https://doi.org/10.1007/s00125-017-4294-3

31. Erener S, Marwaha A, Tan R, Panagiotopoulos C, Kieffer TJ (2017) Profiling of circulating microRNAs in children with recent onset of type 1 diabetes. JCI Insight 2(4):e89656. https://doi.org/10. 1172 jci.insight. 89656

32. Kratzsch J, Deimel A, Galler A, Kapellen T, Klinghammer A, Kiess W (2004) Increased serum soluble leptin receptor levels in children and adolescents with type 1 diabetes mellitus. Eur J Endocrinol 151(4):475-481. https://doi.org/10.1530/eje.0.1510475

33. Kratzsch J, Knerr I, Galler A et al (2006) Metabolic decompensation in children with type 1 diabetes mellitus associated with increased serum levels of the soluble leptin receptor. Eur J Endocrinol 155(4):609-614. https://doi.org/10.1530/eje.1.02261

34. Zhao S, Li T, Li J et al (2016) miR-23b-3p induces the cellular metabolic memory of high glucose in diabetic retinopathy through a SIRT1-dependent signalling pathway. Diabetologia 59(3):644654. https://doi.org/10.1007/s00125-015-3832-0

35. Grieco FA, Sebastiani G, Juan-Mateu J et al (2017) MicroRNAs miR-23a-3p, miR-23b-3p, and miR-149-5p regulate the expression of proapoptotic BH3-only proteins DP5 and PUMA in human pancreatic $\beta$-cells. Diabetes 66(1):100-112. https://doi.org/10. 2337/db16-0592

36. van de Bunt M, Gaulton KJ, Parts L et al (2013) The miRNA profile of human pancreatic islets and beta-cells and relationship to type 2 diabetes pathogenesis. PLoS One 8(1):e55272. https://doi.org/10. 1371/journal.pone.0055272

37. Platania CBM, Maisto R, Trotta MC et al (2019) Retinal and circulating miRNA expression patterns in diabetic retinopathy: an in silico and in vivo approach. Br J Pharmacol 176(13):2179-2194. https://doi.org/10.1111/bph.14665

38. Zampetaki A, Willeit P, Burr S et al (2016) Angiogenic microRNAs Linked to Incidence and Progression of Diabetic Retinopathy in Type 1 Diabetes. Diabetes 65(1):216-227. https://doi.org/10. 2337/db15-0389
39. Cho S, Wu CJ, Yasuda $\mathrm{T}$ et al (2016) miR-23 27 24 clusters control effector $\mathrm{T}$ cell differentiation and function. J Exp Med 213(2):235-249. https://doi.org/10.1084/jem.20150990

40. Torri A, Carpi D, Bulgheroni E et al (2017) Extracellular microRNA signature of human helper $\mathrm{T}$ cell subsets in health and autoimmunity. J Biol Chem. https://doi.org/10.1074/jbc.M116. 769893

41. de Jong VM, van der Slik AR, Laban S et al (2016) Survival of autoreactive $\mathrm{T}$ lymphocytes by microRNA-mediated regulation of apoptosis through TRAIL and Fas in type 1 diabetes. Genes Immun 17(6):342-348. https://doi.org/10.1038/gene.2016.29

42. Fordham JB, Naqvi AR, Nares S (2015) miR-24 regulates macrophage polarization and plasticity. J Clin Cell Immunol 6(5):362370. https://doi.org/10.4172/2155-9899.1000362

43. Pua HH, Steiner DF, Patel S et al (2016) MicroRNAs 24 and 27 suppress allergic inflammation and target a network of regulators of $\mathrm{T}$ helper 2 cell-associated cytokine production. Immunity 44(4): 821-832. https://doi.org/10.1016/j.immuni.2016.01.003

44. Marabita F, de Candia P, Torri A, Tegner J, Abrignani S, Rossi RL (2015) Normalization of circulating microRNA expression data obtained by quantitative real-time RT-PCR. Brief Bioinform 17(2):204-212. https://doi.org/10.1093/bib/bbv056

45. Fortunato O, Boeri M, Verri C et al (2014) Assessment of circulating microRNAs in plasma of lung cancer patients. Molecules 19(3): 3038-3054. https://doi.org/10.3390/molecules19033038

46. El-Asrar MA, Adly AA, Ismail EA (2012) Soluble CD40L in children and adolescents with type 1 diabetes: relation to microvascular complications and glycemic control. Pediatr Diabetes 13(8):616624. https://doi.org/10.1111/j.1399-5448.2012.00881.x

47. Pham MN, Kolb H, Mandrup-Poulsen T et al (2013) Serum adipokines as biomarkers of beta-cell function in patients with type 1 diabetes: positive association with leptin and resistin and negative association with adiponectin. Diabetes Metab Res Rev 29(2):166170. https://doi.org/10.1002/dmrr.2378

48. Saeed FA, Castle GE (1998) Neutrophil chemiluminescence during phagocytosis is inhibited by abnormally elevated levels of acetoacetate: implications for diabetic susceptibility to infections. Clin Diagn Lab Immunol 5(5):740-743. https://doi.org/10.1128/ CDLI.5.5.740-743.1998

49. Stewart RJ, Marsden PA (1995) Biologic control of the tumor necrosis factor and interleukin-1 signaling cascade. Am J Kidney Dis 25(6):954-966. https://doi.org/10.1016/0272-6386(95)905820

50. Simonet WS, Lacey DL, Dunstan CR et al (1997) Osteoprotegerin: a novel secreted protein involved in the regulation of bone density. Cell 89(2):309-319. https://doi.org/10.1016/S0092-8674(00) 80209-3

51. Hofbauer LC, Shui C, Riggs BL et al (2001) Effects of immunosuppressants on receptor activator of NF- $\mathrm{KB}$ ligand and osteoprotegerin production by human osteoblastic and coronary artery smooth muscle cells. Biochem Biophys Res Commun 280(1): 334-339. https://doi.org/10.1006/bbrc.2000.4130

52. Van Campenhout A, Golledge J (2009) Osteoprotegerin, vascular calcification and atherosclerosis. Atherosclerosis 204(2):321-329. https://doi.org/10.1016/j.atherosclerosis.2008.09.033

53. Tsentidis C, Gourgiotis D, Kossiva L et al (2016) Higher levels of sRANKL and osteoprotegerin in children and adolescents with type 1 diabetes mellitus may indicate increased osteoclast signaling and predisposition to lower bone mass: a multivariate cross-sectional analysis. Osteoporos Int 27(4):1631-1643. https://doi.org/10. 1007/s00198-015-3422-5

54. Grzelka A, Naskret D, Araszkiewicz A, Uruska A, Wegner M, Zozulinska-Ziolkiewicz D (2017) Higher concentrations of osteoprotegerin in type 1 diabetic patients are related to retinopathy: Results from the Poznan Prospective Study. Adv Clin Exp Med 26(9):1343-1349. https://doi.org/10.17219/acem/65072 
55. Chrysis D, Efthymiadou A, Mermigka A, Kritikou D, Spiliotis BE (2017) Osteoprotegerin, RANKL, ADMA, and fetuin-A serum levels in children with type I diabetes mellitus. Pediatr Diabetes 18(4):277-282. https://doi.org/10.1111/pedi.12384

56. Elsamahy MH, Elhenawy YI, Nawar MM (2015) Plasma osteoprotegerin concentrations in type 1 diabetic patients with albuminuria. J Diabetes Complicat 29(4):563-567. https://doi.org/10.1016/j. jdiacomp.2015.02.008

57. Loureiro MB, Ururahy MA, Freire-Neto FP et al (2014) Low bone mineral density is associated to poor glycemic control and increased OPG expression in children and adolescents with type 1 diabetes. Diabetes Res Clin Pract 103(3):452-457. https://doi.org/10.1016/j. diabres.2013.12.018

58. Gordin D, Soro-Paavonen A, Thomas MC et al (2013) Osteoprotegerin is an independent predictor of vascular events in Finnish adults with type 1 diabetes. Diabetes Care 36(7):18271833. https://doi.org/10.2337/dc12-2170

59. Grauslund J, Rasmussen LM, Green A, Sjolie AK (2010) Does osteoprotegerin relate to micro- and macrovascular complications in long-term type 1 diabetes? Scand J Clin Lab Invest 70(3):188193. https://doi.org/10.3109/00365511003653599

60. Rasmussen LM, Tarnow L, Hansen TK, Parving HH, Flyvbjerg A (2006) Plasma osteoprotegerin levels are associated with glycaemic status, systolic blood pressure, kidney function and cardiovascular morbidity in type 1 diabetic patients. Eur J Endocrinol 154(1):7581. https://doi.org/10.1530/eje.1.02049

61. Terrazzano G, Bruzzaniti S, Rubino V et al (2020) Type 1 diabetes progression is associated with loss of $\mathrm{CD}^{+} \mathrm{CD} 56^{+}$regulatory $\mathrm{T}$ cells that control $\mathrm{CD} 8^{+} \mathrm{T}$-cell effector functions. Nature Metabolism 2(2):142-152. https://doi.org/10.1038/s42255-0200173-1

62. Wang K, Yuan Y, Cho JH, McClarty S, Baxter D, Galas DJ (2012) Comparing the microRNA spectrum between serum and plasma. PLoS One 7(7):e41561. https://doi.org/10.1371/journal.pone. 0041561

Publisher's note Springer Nature remains neutral with regard to jurisdictional claims in published maps and institutional affiliations. 